\title{
Prevalência de constipação intestinal crônica em crianças atendidas em unidade básica de saúde
}

\author{
Prevalence of chronic constipation in children at a primary health care unit \\ Ieda Regina Lopes Del Ciampo ${ }^{1}$, Lívia Carvalho Galvão ${ }^{2}$, \\ Luiz Antônio Del Ciampo ${ }^{3}$, Maria Inez Machado Fernandes ${ }^{4}$
}

\section{Resumo} nica funcional na infância, em uma unidade básica de saúde, e observar as suas principais características clínicas nessa população. Casuística e métodos: 313 crianças, pacientes da unidade básica de saúde Centro de Saúde Escola de Vila Tibério, Ribeirão Preto (SP), com idades entre 1 a 10 anos incompletos, compuseram a amostra. Destas, foram selecionadas 84 constipadas por critérios instituídos pelos autores. O teste do qui-quadrado foi aplicado para a comparação entre os grupos de constipados e não constipados (nível de significância = 0,01)

Resultados: a prevalência de constipação intestinal crônica funcional foi de 26,8\%. Das crianças constipadas, $85,7 \%$ tinham fezes endurecidas, $25 \%$ volume fecal diminuído, $17,9 \%$ intervalos às evacuações maiores que 2 dias (todas as crianças que apresentavam intervalo maior que 2 dias eram constipadas), $14,3 \%$ escape fecal, e 21,4\% sangramento às evacuações.

Conclusões: a prevalência de constipação intestinal foi elevada, tante para a seleção das crianças constipadas.

J Pediatr (Rio J) 2002;78(6):497-502: constipação intestinal, puericultura, hábito intestinal.
Objetivos: estudar a prevalência de constipação intestinal crôe o intervalo entre as evacuações foi um critério diagnóstico impor-

\begin{abstract}
Objective: to study the frequency of chronic constipation in childhood at a Primary Health Care Unit and to observe its characteristics in this population.

Methods: the sample consisted of 313 children aged between one and 10 years treated at the Centro de Saúde Escola, in the district of Tibério, in the town of Ribeirão Preto. Among these, 84 children with chronic constipation were selected. The chi-squared test was used to compare the groups of constipated and nonconstipated children $(\mathrm{p}=0.01)$.

Results: the prevalence of chronic constipation was $26.8 \%$. In the group of chronic constipation, $85.7 \%$ of the children had hardened stools, $25 \%$ showed reduction in fecal mass, $17.9 \%$ had an interval between evacuations longer than 2 days (constipated children), $14.3 \%$ revealed fecal soiling and $21.4 \%$ presented blood in stools.

Conclusion: the prevalence of chronic constipation was high and the interval between evacuations was as an important diagnostic criterion for the selection of constipated children.
\end{abstract}

J Pediatr (Rio J) 2002;78(6):497-502: chronic constipation, child health care, bowel habit.

\section{Introdução}

A constipação intestinal é um problema freqüente na

1. Professora do Curso de Medicina da Univ. de Ribeirão Preto-UNAERP. Faculdade de Medicina de Ribeirão Preto - USP.

3. Professor Doutor do Departamento de Puericultura e Pediatria da Faculdade de Medicina de Ribeirão Preto - USP.

4. Professora Associada do Departamento de Puericultura e Pediatria da Faculdade de Medicina de Ribeirão Preto - USP.

Artigo submetido em 24.01.02, aceito em 14.08.02. infância, e constitui-se da queixa principal em $3 \%$ das consultas pediátricas de rotina ${ }^{1}$. Na infância, a maior causa de constipação é a crônica funcional, compondo $95 \%$ de todos os $\operatorname{casos}^{2}$, e sua prevalência comunitária tem merecido maior preocupação por parte dos pesquisadores na última década, principalmente no nosso país ${ }^{3-6}$, onde pas- 
sou-se a observar um aumento de publicações científicas a esse respeito, denotando prevalência elevada, variando de $14,7 \%$ a $36,5 \%$. A questão da prevalência de constipação infantil em nível comunitário foi explorada com maior profundidade na literatura médica brasileira do que na internacional, conforme enfatizado em recente revisão sobre constipação intestinal na infância ${ }^{7}$. As pesquisas internacionais dedicadas à prevalência da constipação na população pediátrica ainda são em menor número, e as taxas variam de 15 a $34 \%{ }^{8,9}$. A ampla variação das elevadas prevalências encontradas nos diferentes estudos comunitários deve-se, possivelmente, à diversidade de critérios estabelecidos para caracterização da constipação pelos diferentes autores.

Nos últimos anos, principalmente, surgiram diversas definições de constipação intestinal, procurando aumentar a especificidade do seu diagnóstico precoce. A Sociedade Brasileira de Gastroenterologia Pediátrica e Nutrição (SBGPN), em 1984, definiu a constipação intestinal como uma síndrome que consiste na eliminação, com esforço, de fezes ressecadas, ou de consistência aumentada, independentemente do intervalo de tempo entre as evacuações ${ }^{10}$.

Mais recentemente, a Sociedade Americana de Gastroenterologia Pediátrica e Nutrição elaborou a definição de constipação intestinal como o retardo, ou dificuldade nas defecações, presentes por duas ou mais semanas, e suficiente para causar desconforto significante para o paciente ${ }^{11}$.

Atualmente, um grupo de trabalho internacional considerou a constipação intestinal como um sintoma, definido pela ocorrência de qualquer uma das seguintes manifestações, independentemente do intervalo entre as evacuações: eliminação de fezes duras ou em cíbalos, na forma de seixos ou cilíndricas com rachaduras, dificuldade ou dor para evacuar, eliminação esporádica de fezes muito volumosas que entopem o vaso sanitário, ou freqüência de evacuações inferior a 3 por semana, exceto em crianças em aleitamento natural exclusivo ${ }^{12}$.

Levando-se em consideração que em 1996, período em que foi realizada a coleta de dados do presente estudo, as publicações nacionais que caracterizavam os critérios de constipação intestinal estavam apenas emergindo, os autores também criaram os seus próprios, procurando levar em consideração a definição da SBPGN. O presente estudo teve como objetivo estudar a prevalência de constipação intestinal crônica funcional na população infantil de uma unidade básica de saúde e observar suas principais características clínicas.

\section{Métodos}

O estudo foi realizado no Centro de Saúde Escola de Vila Tibério, uma unidade básica de saúde da Faculdade de Medicina de Ribeirão Preto da Universidade de São Paulo, em Ribeirão Preto - SP, no período de março/96 a fevereiro/97.
Trata-se de um estudo transversal descritivo, de prevalência, com entrevista estruturada, respondida pelos responsáveis que acompanhavam as crianças. A entrevista foi parcialmente adaptada de um questionário aplicado em estudo realizado com alunos de medicina, os quais respondiam eles próprios às questões ${ }^{13}$.

Através do programa Epi-Info - versão 6, foi calculada $\mathrm{e}$, posteriormente, selecionada uma amostra representativa de $24 \%$ da população (313 crianças), com idade entre 1 ano completo e 10 anos incompletos, que apresentava registro ativo e havia freqüentado o centro de saúde nos doze meses que antecederam a entrevista (1.390 crianças).

As entrevistas foram realizadas por apenas um entrevistador, que utilizou quatro períodos da semana (segundas a sextas-feiras, matutino ou vespertino) para a realização das entrevistas, sendo que estes períodos eram sorteados semanalmente, a fim de não provocar um viés, pensando-se que indivíduos constipados poderiam procurar a unidade de saúde nos dias de coleta dos dados, quando passassem a saber que havia um estudo sobre constipação no local.

As crianças incluídas no estudo preencheram os seguintes critérios: devia haver consentimento do responsável para inclusão no estudo, morar na área de abrangência da unidade de saúde, apresentar registro nesta unidade de saúde, ter havido três ou mais consultas médicas de rotina no local, estar em bom estado geral no momento da entrevista, estar acompanhada pela mãe, ou pessoa que convivesse com a criança na maior parte do dia, haver ausência de doenças ou medicações que predispusessem à constipação intestinal.

A amostra estudada foi dividida em dois grupos (constipados e não constipados), de acordo com critérios definidos de constipação estabelecidos pelos autores (Tabela 1), levando-se em consideração a escassez de estudos definindo critérios para o estabelecimento da constipação intestinal em nosso meio, no momento da coleta de dados.

Aplicou-se o teste do qui-quadrado $\left(\chi^{2}\right)$, para a comparação entre os dois grupos (nível de significância $=0,01$ ), e o teste exato de Fisher, na impossibilidade de aplicação do teste anterior.

O projeto foi analisado e aprovado pela comissão de ética do Centro de Saúde Escola do Ipiranga de Ribeirão Preto, tendo o seu parecer favorável em fevereiro/96.

\section{Resultados}

A mediana para idade das 313 crianças estudadas foi 4 anos, e não houve diferença em relação à idade entre os 84 constipados (mediana 3,5 anos; 1-10) e os 229 não constipados (mediana 4,0 anos; 1-10).

A prevalência de constipação intestinal crônica foi igual a $26,8 \%$ (84/313), sendo $27,6 \%$ do sexo feminino e $26,1 \%$ do masculino ( $\mathrm{p}>0,01)$. 
Tabela 1 - Critérios estabelecidos para o diagnóstico de constipação intestinal crônica funcional

\begin{tabular}{ll}
\hline Critérios de constipação intestinal crônica funcional \\
Sinais maiores & Sinais menores \\
Fezes cilíndricas ressecadas & Volume aumentado \\
Fezes fragmentadas & Intervalo entre as evacuações maior ou igual a 2 dias \\
Eliminação dolorosa & Sangramento \\
Eliminação com esforço & Demora para iniciar a evacuação \\
Escape fecal &
\end{tabular}

Constipados: 2 ou mais critérios maiores, ou 1 maior acompanhado de 2 menores, presentes há mais de 1 mês.

Os resultados obtidos em relação às características das evacuações estão compilados na Tabela 2.

Inicialmente as variáveis "fezes cilíndricas ressecadas" (44\% constipados; $11,9 \%$ não constipados) e "fezes fragmentadas" (41,7\% constipados; $8,7 \%$ não constipados) foram analisadas separadamente, não havendo diferença significante entre os dois grupos, porém, quando foram aglutinadas em uma única variável, denominada "fezes endurecidas" (cilíndricas ressecadas ou fragmentadas), ela estava presente em $85,7 \%$ (72/84) das crianças constipadas.

Para as 84 crianças constipadas, foi inquirida a idade de início da constipação intestinal, sendo que em 30 (46,9\%) a constipação intestinal teve início antes de um ano de idade.

\section{Discussão}

Os resultados desta pesquisa apontam para uma prevalência de constipação intestinal crônica funcional (CICF) igual a $26,8 \%$, inserindo-se na ampla variação de 2 a 36,5\% encontrada por outros autores ${ }^{3-6,8,13,16,17}$ que utilizam metodologia diversificada, tanto no que diz respeito à forma de obtenção dos dados, quanto na seleção da população investigada. O presente estudo inclui-se no conjunto de trabalhos que abordam a prevalência de constipação intestinal em grupos específicos de crianças, compondo as suas amostras em unidades básicas de saúde, ambulatórios de pediatria geral ou instituições de ensino, populações estas consideradas representativas da população pediátrica, sendo sua grande maioria publicados a partir de 1988. A prevalência de CICF, no presente estudo, é próxima à de $25 \%$ encontrada no Ambulatório de Pediatria Geral da Faculdade de Medicina de Botucatu ${ }^{4}$, e pouco mais elevada que a de 20,6\% encontrada no Ambulatório de Pediatria do Hospital Materno Infantil Presidente Vargas ${ }^{15}$ (252 crianças sem queixas do aparelho digestivo), que pode ter ocorrido devido ao quesito "ausência de sintomas digestivos" não fazer parte dos critérios de inclusão. A maior prevalência, de 36,6\%, observada em ambulatórios do INAMPS e do Hospital de Clínicas de Porto Alegre-RS, pode ter sido devida a diferenças na conceituação da constipação intestinal (os autores não especificaram os critérios utilizados para o diagnóstico de CICF), ou pela própria variação local. Prevalências elevadas de constipação em escolas de periferia de Botucatu foram de $35,5 \%$ e $29 \%$ (pelos critérios brando e rigoroso, respectivamente, utilizados pelos autores $)^{4}$ são pertinentes, pois parte da população doente não procura por atendimento médico em unidade básica de saúde e, portanto, não poderia ser diagnosticada nos estudos realizados neste local. A prevalência de $12 \%$ nos CIEPS da Ilha do Governador, RJ, em crianças de 8 a 10 anos de idade 6 , pode refletir a realidade do local e/ou da estreita faixa etária estudada. A prevalência de $17,3 \% \mathrm{em}$ comunidade de baixa renda, em Recife, pode também refletir a realidade local e/ou a diferença nos critérios utilizados (complicações da CICF não foram utilizadas como critério diagnóstico $)^{5}$.

Nos EUA, a prevalência de constipação intestinal igual a $2 \%$ em estudo epidemiológico (levantamento por entrevistas domiciliares, registros nacionais, hospitalares, ambulatoriais e estatísticos vitais ${ }^{16}$ pode ter sido subestimada, pois os registros utilizados poderiam não ter o diagnóstico correto, se os indivíduos se referissem apenas às suas complicações.

$\mathrm{Na}$ análise da consistência das fezes, observou-se que, provavelmente, os critérios utilizados nesse estudo foram por demais rigorosos, quando desmembrados os quesitos 
Tabela 2 - Características das evacuações das 313 crianças entrevistadas no CSE Vila Tibério, no período de março/96 a fevereiro/97, para avaliação da prevalência de constipação intestinal crônica funcional

\begin{tabular}{|c|c|c|c|}
\hline \multirow{2}{*}{ Critérios avaliados } & & \multicolumn{2}{|c|}{ Grupos } \\
\hline & & Constipados & Não constipados \\
\hline \multirow[t]{4}{*}{ Consistência das fezes } & Ressecada (cilíndrica ressecada/fragmentada) & $72(85,7 \%) \dagger$ & $45(19,7 \%)$ \\
\hline & Normal (pastosa) & $10(11,9 \%)$ & $146(63,7 \%) \dagger$ \\
\hline & Amolecida (semi-líquida/líquida) & $2(4,2 \%)$ & $28(12,2 \%)$ \\
\hline & Não sabe* & $0(0 \%)$ & $10(4,4 \%)$ \\
\hline \multirow[t]{4}{*}{ Volume das fezes } & Aumentado & $17(20,2 \%) \dagger$ & $15(6,6 \%)$ \\
\hline & Normal & $46(54,8 \%)$ & $190(83 \%) \dagger$ \\
\hline & Diminuído & $21(25 \%) \dagger$ & $12(5,2 \%)$ \\
\hline & Não sabe* & $0(0 \%)$ & $12(5,2 \%)$ \\
\hline \multirow{3}{*}{$\begin{array}{l}\text { Intervalo entre } \\
\text { as evacuações }\end{array}$} & $<2$ dias & $69(82,1 \%)$ & $227(99,1 \%)$ \\
\hline & $\geq 2$ dias & $15(17,9 \%) \ddagger$ & $0(0 \%)$ \\
\hline & Não sabe* & $0(0 \%)$ & $2(0,9 \%)$ \\
\hline \multirow[t]{2}{*}{ Sangramento anal } & $\operatorname{sim}$ & $18(21,4 \%) \ddagger$ & $1(0,4 \%)$ \\
\hline & não & $66(78,6 \%)$ & $228(99,6 \%)$ \\
\hline \multirow[t]{2}{*}{ Escape fecal } & $\operatorname{sim}$ & $12(14,3 \%) \ddagger$ & $3(1,3 \%)$ \\
\hline & não & $72(85,7 \%)$ & $226(98,7 \%)$ \\
\hline \multirow[t]{2}{*}{ Dor à evacuação } & $\operatorname{sim}$ & $42(50 \%) \ddagger$ & $0(0 \%)$ \\
\hline & não & $42(50 \%)$ & $229(100 \%)$ \\
\hline \multirow[t]{2}{*}{ Esforço às evacuações } & $\operatorname{sim}$ & $77(91,6 \%) \dagger$ & $7(3 \%)$ \\
\hline & não & $7(8,4 \%)$ & $222(97 \%)$ \\
\hline
\end{tabular}

$\dagger \chi^{2}(\mathrm{p}<0,01) . \quad \neq$ Teste exato de Fisher $(\mathrm{p}<0,01)$.

* obs: em algumas questões havia falta de conhecimento por alguns responsáveis, porém sem prejuízo aos critérios de seleção instituídos ou à análise dos dados.

referentes à característica "fezes de consistência aumentada" em fezes cilíndricas ressecadas e fragmentadas. Dessa maneira, a freqüência encontrada entre os 2 grupos não foi significante. Entretanto, quando esses dois quesitos foram agrupados, a alta freqüência de crianças constipadas $(85,7 \%)$, com fezes de consistência aumentada ( $44 \%$ cilíndricas ressecadas e $41,7 \%$ fragmentadas), tornou-se significante, concordando com autora que encontrou $66,6 \%$ $(69,5 \% \mathrm{Me} 63,7 \% \mathrm{~F})$ das 330 crianças constipadas (critério rigoroso), com eliminação de cíbalos às evacuações, em estudo realizado com 1.145 crianças estudantes de 5 escolas do ciclo básico localizadas na periferia da cidade de Botucatu-SP 4 . Isto demonstra que o aspecto "fezes de consistência aumentada" (fezes cilíndricas ressecadas + fragmentadas) teria sido um critério mais adequado para o diagnóstico de CICF. O agrupamento de ambos os critérios em questão não invalidaria os resultados, pois, além de serem excludentes, eram critérios maiores, e a classificação de constipação intestinal crônica (CIC) consistia na presença de 1 sinal maior e 2 menores ou 2 sinais maiores.

A frequiência significante de crianças com volume fecal aumentado concorda com estudos de revisão que referem alta prevalência de fezes volumosas nas crianças portadoras de CIC. Já a elevada freqüência de crianças constipadas com volume fecal diminuído pode corresponder à eliminação incompleta do conteúdo fecal (evacuações parciais), que ocorre em algumas crianças constipadas ${ }^{10}$.

Todas as crianças que evacuavam em intervalo maior ou igual a 2 dias eram constipadas, portanto, a frequêencia determinada foi um critério importante para a classificação da CICF. Este achado permite sugerir modificações nos critérios diagnósticos de CICF adotados nesta pesquisa, colocando "freqüência maior ou igual a 2 dias" como 
critério maior. Não foram encontrados estudos de constipação em comunidades ou unidades básicas de saúde que tivessem observado o intervalo entre as evacuações, ficando impossibilitada a sua comparação.

As frequiências das alterações relativas às eliminações das fezes (escape fecal, sangramento, etc.) nos ambulatórios de pediatria geral concordam com as deste estudo. Encontrou-se escape fecal em 11,5\% de 252 crianças, 1 mês a 15 anos de idade, no ambulatório de Pediatria do Hospital Materno Infantil Presidente Vargas ${ }^{15}$, próximo aos 14,3\% observados neste estudo. Dor ao evacuar ocorreu em $33,1 \%$ dos meninos e $41,9 \%$ das meninas constipados, estudantes do ciclo básico da cidade de Botucatu-SP, menor que o encontrado nesta pesquisa. Sangramento ocasional às evacuações ocorreu em 12,6\% no sexo masculino e em 19,6\% no feminino, também menores que os encontrados neste estudo. Entretanto a freqüência de escape fecal (30,5\% masculino e $31,8 \%$ feminino) foi maior que os $14,3 \%$ aqui encontrados. Estas diferenças talvez sejam explicadas pela faixa etária das crianças estudadas, já que as de mais tenra idade apresentam alterações na eliminação das fezes sem escape fecal, que se estabelece a partir dos 4 anos de idade, como encontrado nas crianças estudadas pelos autores, em que a mediana para idade era igual a 8 anos e 4 meses $^{4}$. A prevalência de escape fecal foi igual a 2,6\% em estudo comunitário realizado nos CIEPS da Ilha do GovernadorRJ, com 391 crianças, de 8 a 10 anos de idade ${ }^{6}$, resultado semelhante aos 5\% (considerando-se a população total) aqui detectados.

A elevada freqüência de início da constipação intestinal antes de um ano de idade concorda com os 67,8\% de início dos sintomas de constipação já no primeiro ano de vida, encontrados por alguns autores ${ }^{18}$, mas discorda da maioria dos estudos que relatam maior frequiência de início da constipação após um ano de idade, atingindo o máximo entre dois e três anos ${ }^{19,20}$. Cabe aqui ressaltar que a técnica recordatória utilizada é sujeita a erros, uma vez que não se tratava de um estudo prospectivo, sendo necessários novos estudos para sua validação.

De acordo com os resultados encontrados, a abordagem do conjunto de alterações relacionadas à eliminação das fezes determinando um critério para o diagnóstico da constipação intestinal crônica funcional mostrou-se adequada para a avaliação de sua prevalência na amostra estudada.

\section{Referências bibliográficas}

1. Loenning-Baucke V. Chronic constipation in children. Gastroenterology 1993;105:1557-64.

2. Loenning-Baucke V. Encopresis and soiling [review]. Pediatr Clin North Am 1996;43:279-98.
3. Zaslavsky C, Ávila EL, Araújo MA, Pontes MN, Lima NE. Constipação intestinal na infância - um estudo de prevalência. $\mathrm{R}$ AMRIGS 1988;32:100-2.

4. Maffei HVL, Moreira FL, Oliveira WM, Sanini V. Prevalência de constipacão intestinal em escolares do ciclo básico. J Pediatr (Rio J) 1997;73:340-4.

5. Motta MEFA, Silva GAP. Constipação intestinal crônica na infância: diagnóstico e prevalência em uma comunidade de baixa renda. J Pediatr (Rio J) 1998;74:451-4.

6. Araújo Sant'Anna AM, Calçado AC. Constipation in schoolaged children at public schools in Rio de Janeiro, Brazil. J Pediatr Gastroenterol Nutr 1999;29:190-3.

7. Morais MB, Maffei HVL. Constipação intestinal. J Pediatr (Rio J) 2000;76 (Supl 1):147-56.

8. Issenman RM, Hewson S, Pirhonen D, Taylor W, Tirosh A. Are chronic complaints the result of abnormal dietary patterns? Am J Dis Child 1987;141:679-82.

9. Roma E, Adamidis D, Nikolara R, Constantopoulos A, Messaritakis J. Diet and chronic constipation in children: the role of fiber. J Pediatr Gastroenterol Nutr 1999;28:169-74.

10. Loening-Baucke V. Chronic constipation in children. Gastroenterology 1993;105:1557-64. Sociedade Paulista de Gastroenterologia Pediátrica e Nutrição (SPGPN). Boletim n ${ }^{\circ} 4$; 1994.

11. Baker SS, Liptak GS, Colleti RB, Croffie JM, Di Lorenzo C, Ector W, et al. Constipation in infants and children: evaluation and treatment. A medical position statement of North American Society for Pediatric Gastroenterology and Nutrition. J Ped Gastroenterol Nutr 1999;29:612-26.

12. Maffei HVL, Morais MB. Constipação intestinal crônica funcional. In: Baebieri D, Palma D, editores. Gastroenterologia e Nutrição. $1^{\mathrm{a}}$ ed. São Paulo-Rio: Atheneu; 2001.p.63-80.

13. Galvão LC, Maffei HL, Suarez VM, Calva RR, Fernandes MIM. Constipação intestinal crônica em estudantes de medicina de Ribeirão Preto. Estudo multicêntrico. In: XII Congresso latinoamericano de gastroenterologia pediátrica e nutrição. II Congresso ibero-latino-americano de gastroenterologia pediátrica e nutrição. São Paulo; 1996:15-9.

14. Sant'anna A. Constipação intestinal no Ambulatório de Gastroenterologia Pediátrica da Universidade Federal do Rio de Janeiro. In: XII Congresso latino-americano de gastroenterologia pediátrica e nutrição. II Congresso ibero-latino-americano de gastroenterologia pediátrica e nutrição, São Paulo; 1996:155.

15. Berti MR, Rech A. Hábitos intestinais em crianças sem queixas do aparelho digestivo. Revista Científica da AMECS 1997;6: 121-4.

16. Sonnenberg A, Koch TR. Epidemiology of constipation in the United States. Dis Col \& Rect 1989;32:1-8.

17. Sandler RS, Jordan MC, Shelton BJ. Demographic and dietary determinants of constipation in the US population. Am J Public Health 1990;80:185-9.

18. Maffei HVL, Moreira FL, Kissimoto M, Chaves SMF, El Faro S, Aleixo AM. História clínica e alimentar de crianças atendidas em ambulatório de gastroenterologia pediátrica (GEP) com constipação intestinal crônica funcional (CICF) e suas possíveis complicações. J Pediatr (Rio J) 1994;70:280-6.

18. Cruz AS, Dias CZ, Zella MJ, Tarastchuck ARV. Características clínicas de 303 crianças portadoras de constipação crônica e resposta ao tratamento clínico. In: XII Congresso latino-americano de gastroenterologia pediátrica e nutrição. II Congresso iberolatino-americano de gastroenterologia pediátrica e nutrição. São Paulo; 1996:15-9. 
19. Zaslavsky C, Pires ALG, Berti MR, Ruaro R, Alles YCJ. Constipação intestinal na infância. In:XII Congresso latinoamericano de gastroenterologia pediátrica e nutrição. II Congresso ibero-latino-americano de gastroenterologia pediátrica e nutrição. São Paulo;1996:15-9.
Endereço para correspondência

Dra. Ieda Regina Lopes Del Ciampo

Avenida Abade Constantino, 371

CEP 14040-290 - Ribeirão Preto, SP

E-mail:irlopes@keynet.com.br 